\title{
CONTAMINATION OF BROADBAND PHOTOMETRY BY NEBULAR EMISSION IN HIGH-REDSHIFT GALAXIES: INVESTIGATIONS WITH KECK'S MOSFIRE NEAR-INFRARED SPECTROGRAPH
}

\author{
Matthew A Schenker ${ }^{1}$, Richard S Ellis ${ }^{1}$, Nick P Konidaris $^{1}$, and Daniel P Stark ${ }^{2,3}$ \\ ${ }^{1}$ Department of Astrophysics, California Institute of Technology, MC 249-17, Pasadena, CA 91125, USA; schenker@astro.caltech.edu \\ 2 Department of Astronomy and Steward Observatory, University of Arizona, Tucson, AZ 85721, USA \\ Received 2013 June 6; accepted 2013 August 31; published 2013 October 17
}

\begin{abstract}
Earlier work has raised the potential importance of nebular emission in the derivation of the physical characteristics of high-redshift Lyman break galaxies. Within certain redshift ranges, and especially at $z \simeq 6-7$, such lines may be strong enough to reduce estimates of the stellar masses and ages of galaxies compared with those derived assuming the broadband photometry represents stellar light alone. To test this hypothesis at the highest redshifts where such lines can be probed with ground-based facilities, we examine the near-infrared spectra of a representative sample of $283.0<z<3.8$ Lyman break galaxies using the newly commissioned MOSFIRE near-infrared spectrograph at the Keck I telescope. We use these data to derive the rest-frame equivalent widths (EWs) of [O III] emission and show that these are comparable with estimates derived using the spectral energy distribution (SED) fitting technique introduced for sources of known redshift by Stark et al. Although our current sample is modest, its [O III] EW distribution is consistent with that inferred for $\mathrm{H} \alpha$ based on SED fitting of Stark et al.'s larger sample of $3.8<z<5$ galaxies. For a subset of survey galaxies, we use the combination of optical and near-infrared spectroscopy to quantify kinematics of outflows in $z \simeq 3.5$ star-forming galaxies and discuss the implications for reionization measurements. The trends we uncover underline the dangers of relying purely on broadband photometry to estimate the physical properties of high-redshift galaxies and emphasize the important role of diagnostic spectroscopy.
\end{abstract}

Key words: galaxies: evolution - galaxies: high-redshift - galaxies: starburst

Online-only material: color figures

\section{INTRODUCTION}

Detailed photometry of Lyman break galaxies (LBGs) undertaken with the Hubble Space Telescope (HST) and the Spitzer Space Telescope has provided spectral energy distributions (SEDs) for large samples of LBGs in the important redshift range $3<z<7$. These data have been used to derive valuable estimates of the star formation rates (SFRs), stellar masses, and ages (e.g., Stark et al. 2009; Labbé et al. 2010; González et al. 2011). The stellar mass density is a particularly important measure for the epoch just after reionization ended as it provides a key indication of whether yet earlier star formation at $z>6$ is capable of maintaining cosmic reionization (Robertson et al. 2013).

However, many earlier studies (e.g., Schaerer \& de Barros 2009, 2010; Ono et al. 2010; de Barros et al. 2012) have suggested that high-redshift stellar mass densities may be overestimated because the broadband fluxes analyzed may be significantly contaminated by strong nebular emission lines (e.g., [O II], [O III], $\mathrm{H} \alpha$ ). Although this contamination has been well-documented in local star-forming galaxies (e.g., Sargent \& Searle 1970; Krueger et al. 1995; Papaderos et al. 1998) and more recently at moderate redshifts through $H S T$ grism data (Atek et al. 2011), the extent of such contamination is largely unconstrained at the high redshifts relevant for understanding cosmic reionization.

Early attempts to address this problem at high redshift used "forward modeling" techniques that fit stellar population synthesis models, including nebular emission, to broadband photometry without any knowledge of the galaxy redshifts. These methods suffer from a major limitation: it is not known a priori which filters are likely contaminated. In this respect,

\footnotetext{
${ }^{3}$ Hubble Fellow
}

examining the SEDs of galaxies of known spectroscopic redshift provides a much sounder approach (Shim et al. 2011). In Stark et al. (2013), we examined galaxies with known spectroscopic redshifts drawn from our extensive Keck survey to compare SEDs in the redshift range $3.8<z<5.0$, where line contamination is likely, with those in an uncontaminated range, $3.1<z<3.6$. This comparison showed that $\mathrm{H} \alpha$ emission typically contributes $30 \%$ of the flux at $3.6 \mu \mathrm{m}$, from which we derived a rest-frame equivalent width (EW) distribution for $\mathrm{H} \alpha$. Assuming this distribution, we then showed that nebular emission could have a significant impact on the inferred stellar masses of galaxies at $z \simeq 6-7$ where both short wavelength IRAC filters are contaminated by nebular emission ([O III] and $\mathrm{H} \alpha$, respectively).

An additional motivation for the Stark et al. (2013) study was our interest in addressing a puzzle that arose from early measures of the specific SFR (sSFR) for $z>2$. It had been claimed that this quantity does not evolve strongly between $z \simeq 2$ and 7 (e.g., Stark et al. 2009; González et al. 2010), in contrast with numerical simulations (e.g., Davé et al. 2012) that predict that the sSFR should closely match the inflow rate of baryonic gas. In Stark et al. (2013), we used the EW distribution of $\mathrm{H} \alpha$ derived from the Keck sample to show that the sSFR is likely to evolve more rapidly at $z>4$ than previously thought, supporting up to a five-fold increase between $z \simeq 2$ and 7 (see also de Barros et al. 2012; c.f. González et al. 2012). A similar conclusion has recently been presented by Smit et al. (2013) based on SED fitting a small number of bright lensed galaxies at $6.6<z<7.0^{4}$

\footnotetext{
4 Those authors expressed concern that the Stark et al. (2013) sample might be biased toward a spectroscopic sample with strong Ly $\alpha$ emission but this is not the case; indeed, over $50 \%$ of that sample showed no measurable Ly $\alpha$ emission.
} 
In this paper, we take the subject to the next logical stage by verifying directly with near-infrared spectroscopy the impact of nebular emission in the analysis of SEDs for high-redshift LBGs. Prior to the launch of the James Webb Space Telescope, it is not possible to directly examine contamination by $\mathrm{H} \alpha$ emission within the IRAC warm filters. However, in a manner similar to that employed by Stark et al. (2013), we can investigate contamination by $\mathrm{H} \beta$ and [O III] 4959+5007 $\AA$ in the photometric $K_{S}$ band at $2.2 \mu \mathrm{m}$ by studying a representative sample of $3.0<z<3.8$ spectroscopically confirmed LBGs. Our goal is to determine the rest-frame EW distribution of [O III] directly and to compare this, to the extent possible, with that inferred for $\mathrm{H} \alpha$ from the SED-based study of Stark et al. (2013). Such a spectroscopic program is made possible by the arrival at the Keck I telescope of the multi-slit near-infrared spectrograph MOSFIRE (McLean et al. 2012), which offers the advantage of a significant multiplex gain. Using this new instrument, we target a representative sample of LBGs selected to lie within the $3.0<z<3.8$ redshift range in the GOODS-North (GOODS-N) field. We can thus take advantage of Advanced Camera for Surveys (ACS) photometry from the GOODS survey and improved near-infrared photometry from the CANDELS survey. This extensive photometry further means we can directly compare measured [O III] fluxes with those inferred using the SED-based approach.

Throughout this paper, we adopt a $\Lambda$-dominated, flat universe with $\Omega_{\Lambda}=0.7, \Omega_{M}=0.3$, and $H_{0}=70 h_{70} \mathrm{~km} \mathrm{~s}^{-1} \mathrm{Mpc}^{-1}$. All magnitudes in this paper are quoted in the AB system (Oke \& Gunn 1983). We refer to the HST ACS and WFC3/IR filters F435W, F606W, F775W, F850LP, F105W, F125W, and F160W as $B_{435}, V_{606}, i_{775}, z_{850}, Y_{105}, J_{125}$, and $H_{160}$, respectively.

\section{TARGET SELECTION}

\subsection{Photometry}

For our target selection and SED fitting, it was necessary to assemble a full multi-wavelength catalog across the GOODS-N field. For the HST ACS data, we use the publicly available v2.0 GOODS-N mosaics (Giavalisco et al. 2004). For the newly obtained CANDELS WFC3/IR data (Grogin et al. 2011; Koekemoer et al. 2011), we combine the single epoch mosaics publicly available as of 2013 March, weighted by exposure time, using the image combination routine SWARP (Bertin et al. 2002). Our CANDELS reductions comprise the first seven epochs taken in GOODS-N and so a number of objects do not yet have coverage in the $Y_{105}$ filter. However, as we have $J_{125}$ and $H_{160}$ imaging for all targets, this does not constitute a significant weakness for our SED analyses.

To compute accurate colors for each object, we measured the flux of each object in point-spread function (PSF)-matched images. Our PSF matching was done using the ColorPro program (Coe et al. 2006). Briefly, we first assembled a PSF for each filter by shifting and stacking $\sim 20$ bright unsaturated stars. We detected all objects using the $i_{775}$ image and determined colors using matched isophotal apertures after degrading the $i_{775}$ image full width at half maximum (FWHM) to equal that of each other image in turn. Colors were then corrected to total values by computing the offset from MAG AUTO for the $i_{775}$ band in the GOODS v2.0 catalog. In cases where MAG AUTO was deemed to be unreliable by visual inspection of the images, we used the offset to a flux measured in a $1^{\prime \prime}$. 0 diameter aperture to derive a total magnitude.
For the key photometry in the spectral region of interest, we use the $K_{S}$-band image derived from ultra-deep Canada France Hawaii Telescope imaging published by Wang et al. (2010). This image has total exposure time $t=49.4 \mathrm{hr}$ and a $5 \sigma$ limiting magnitude of 24.5 in the GOODS-N field. As the FWHM of the image is $0.7-0 . .8$, we do not attempt to PSF match the other images to this coarser resolution. Rather, as our objects are largely compact, we perform photometry in 1".0 diameter apertures and apply a correction of 0.7 mag for flux falling outside the aperture, determined from analyses of isolated, unsaturated stellar sources in the $K_{S}$ image. Finally, where available, we use deconfused Spitzer 3.6 and $4.5 \mu$ m photometry from the GOODS Superdeep IRAC data. A full description of the deconfusion procedure can be found in McLure et al. (2011).

\subsection{Spectroscopic Sample}

Our primary source of targets consisted of spectroscopically confirmed galaxies from the Keck survey of $3<z<6$ LBGs (Stark et al. 2010, 2011) in the GOODS-N field. Briefly, this sample was compiled via optical follow-up of color-selected LBGs ( $B, V$, and $i$-drops) with the DEIMOS spectrograph (Faber et al. 2003) on Keck II. The relevant observations took place between 2008 and 2010 and integration times for the sample considered here ranged from 5.0 to $7.0 \mathrm{hr}$. The interested reader can find the full details of the sample in Stark et al. (2010). ${ }^{5}$

From this compilation, we chose to undertake near-infrared spectroscopy of LBGs, primarily $B$ drops, with confirmed redshifts $3.0<z<3.8$ since both $\mathrm{H} \beta$ and [O III] $4959+$ 5007 lie within the MOSFIRE $K$-band transmission window. As the original $B$-drop sample only sparsely populates the above redshift range, we increased the available sample via further DEIMOS observations in 2012 June using photometric redshifts to improve the redshift coverage in anticipation of the present needs. To achieve this, we first created a catalog of $B_{435}$ dropout galaxies using the GOODS v2.0 catalogs and the selection criteria outlined in Stark et al. (2009). These SEDs were then evaluated with a photometric redshift code to assess their chances of lying at $z \leqslant 3.8$. This is a key step, as the $B$-drop sample possesses a mean redshift of $z \sim 4.0$ and only $\simeq 25 \%$ of $B$ drops lie below $z=3.8$ (see Figure 1). Our priorities for inclusion of these targets on the mask were the probability of lying at $z<3.8$ and the $z_{850}$ magnitude for each target, with brighter targets favored. The observations and data reduction for these additional targets were undertaken in the same manner as those previously described in Stark et al. (2010).

This investment of spectroscopic observing time enabled us to select targets that are known a priori to lie in the accessible redshift range and maximizes our efficiency. Additionally, in the event of non-detections of nebular emission lines, prior knowledge of the redshift affords robust upper limits on the fluxes. In total, we were able to include 13 out of a total of 23 objects with prior spectroscopic identification at $3.0<z<3.8$ in our MOSFIRE campaign. As in our additional DEIMOS mask, these objects were prioritized by their $z_{850}$ magnitude (brighter galaxies being favored) and our ability to create efficient mask placements. In all tables and plots, these objects with pre-determined DEIMOS redshifts are identified with the tag "-D" following their IDs.

Since we are interested in the nebular emission line properties of this sample, it is important to note that all the targets for

\footnotetext{
5 A final catalog of this extensive spectroscopic survey is now being prepared for publication (D. P. Stark et al., in preparation).
} 


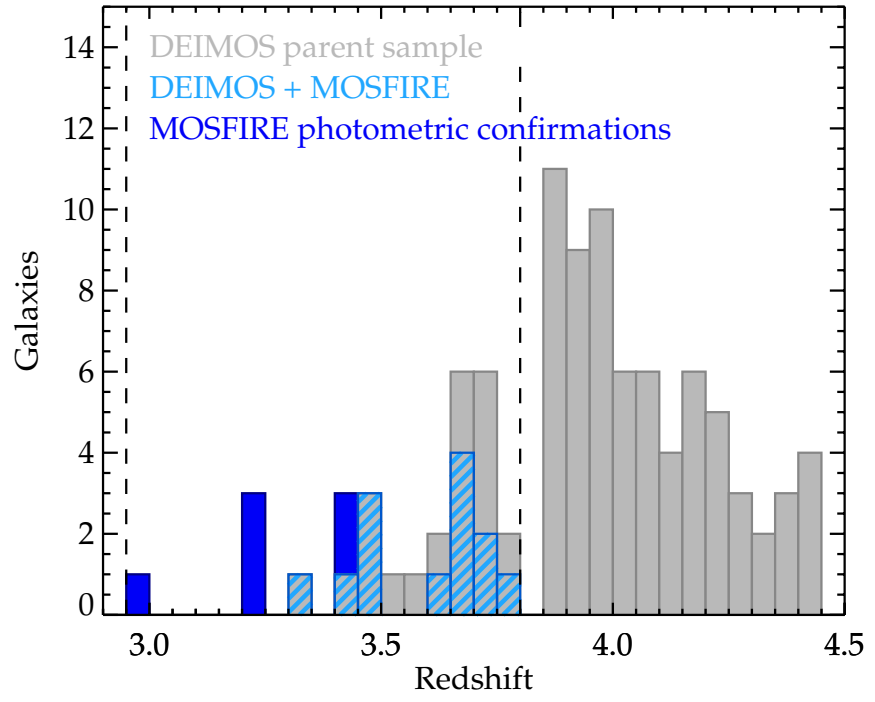

Figure 1. Redshift distribution of LBGs targeted with MOSFIRE. The gray histogram indicates the parent sample within GOODS-N from our prior DEIMOS campaign. The light blue cross-hatched histogram denotes the subset of the DEIMOS spectroscopic sample studied with MOSFIRE and the dark blue histogram denotes those drawn from a photometric selection (see Section 2). The dashed lines show the boundaries within which [O III] lies in the MOSFIRE $K$-band spectral range.

(A color version of this figure is available in the online journal.)

the various DEIMOS campaigns were selected only using ACS photometry and thus should not be significantly biased toward objects with strong nebular emission.

\subsection{Photometric Sample}

As MOSFIRE can accommodate as many as 46 slits on a single mask, we sought to augment our spectroscopic sample above with additional photometrically selected LBGs. Our procedure for adding new targets was largely as described above, except the SEDs now incorporated CANDELS WFC3 data where appropriate and deconfused Spitzer IRAC photometry.

Although one of the goals of this campaign was to verify the technique pioneered in Shim et al. (2011) and Stark et al. (2013) of using photometric excesses to determine line strengths, we specifically avoided prioritizing targets by their $K$-band magnitude. This allows us to construct an unbiased sample and thus a better estimate of the true distribution of nebular line EWs in the following analysis.

In total, we included 15 galaxies from this photometric sample spread across these two masks, but failed to observe any significant line emission in eight of these. Given the uncertainty in the photometric redshift estimates, we consider two extreme case to explain this lack of emission in our later analysis (Section 4.2), namely, that all lie outside the required redshift range $3.0<z<3.8$, or that all lie within it but with line emission fainter than our detection limit.

\section{OBSERVATIONS}

The targets defined above were observed using MOSFIRE (McLean et al. 2012) on the Keck I telescope. We observed two masks on separate observing runs, both using the $K$-band grating. The first mask was observed on the night of 2013 March 20-21 for a total of $4.25 \mathrm{hr}$ integration time, of which $\simeq 1 \mathrm{hr}$ was affected by thin clouds. The average seeing for this mask was 0.75 arcsec FWHM. Our second mask was observed on the night of 2013 April 16-17. We obtained a total of $2.50 \mathrm{hr}$ of useful integration time, including approximately 40 minutes with thin clouds, and an average seeing of 0.70 arcsec FWHM.

We used an $A B A B$ dither pattern with individual exposures of $180 \mathrm{~s}$ and a slit width of 0.7 arcsec for all targets. On each mask, in addition to our science targets, we also included a star with $K_{A B}<19.0$. Using the longslit mode of MOSFIRE, also with a 0.7 arcsec slit, we observed an A0V star at twilight during each of our runs. Flux calibration was performed using two methods. In the first, we use a spectrum of Vega from the HST CALSPEC database, ${ }^{6}$ scaled to the $K_{s}$ magnitude of our A0V standard to determine the sensitivity as a function of wavelength.

In the second method, we use the observations of our standard star to determine the shape of the sensitivity, but set the overall normalization using the star placed on each of our masks. This is done by matching the average flux for the star at wavelengths probed by the $K_{s}$ band to the value measured in the Wang et al. (2010) $K_{s}$ photometry. This method has the advantage of intrinsically accounting for the effects of thin cloud and slit loss due to seeing and pointing error during our observations. Its disadvantage is the lack of knowledge of the spectral type of the calibration star. Reassuringly, the two methods agree to within $10 \%$, so we chose to utilize the second method in order to better account for the cloud encountered during our observations. For our analysis, we assume a $15 \%$ error on the flux calibration in all calculations.

The data were reduced using the publicly available MOSFIRE data reduction pipeline. ${ }^{7}$ Briefly, the pipeline first creates a median, cosmic ray-subtracted flat field image for each mask. Wavelength solutions for each slit are fit interactively for the central pixel in each slit, then propagated outward to the slit edges to derive a full wavelength grid for each slit. The sky background is estimated as a function of wavelength and time using a series of B splines and subtracted from each frame. The $\mathrm{AB}$ frames are differenced, stacked, rectified, and output for use along with inverse variance-weighted images used for error estimation.

To determine the redshift of each source in our MOSFIRE spectra, we fit three Gaussians to the [O III] 4959, 5007, and $\mathrm{H} \beta$ lines simultaneously, ignoring regions contaminated by nearby sky lines. In the spatial direction, we chose the aperture of our one-dimensional (1D) spectra to enclose at least $90 \%$ of the flux from the star on our mask, which resulted in a width of 9 pixels (1".6). For line flux measurements, apertures in the spectral direction were set to extend from the line center to at least 2.5 times our measured dispersion $(\sigma)$, typically resulting in $\sim 10-15$ pixels. Small corrections were made for flux expected to fall outside of these apertures.

In total, we examined 28 targets, 20 of which are now securely established within the $3.0<z<3.8$ redshift range. Of these, 13 had pre-existing optical spectra from DEIMOS and 7 represent new MOSFIRE spectroscopic confirmations determined from our photometric sub-sample (Section 2.3). The remaining 8 galaxies display no detectable line emission in our MOSFIRE spectra (see Section 4.2 for a discussion of these). Table 1 and Figure 1 summarize the salient properties of the final sample.

\footnotetext{
6 http://www.stsci.edu/hst/observatory/cdbs/calspec.html

7 https://code.google.com/p/mosfire/
} 
Table 1

Properties of Observed Targets

\begin{tabular}{|c|c|c|c|c|c|c|c|c|c|c|}
\hline ID & Mask & RA & Dec & $m_{z 850}$ & $z$ & $\begin{array}{c}\text { Mass/ } \\
\left(10^{9} M_{\odot}\right)\end{array}$ & $\begin{array}{c}\mathrm{SFR} \\
\left(M_{\odot} \mathrm{yr}^{-1}\right)\end{array}$ & $E(B-V)$ & $\begin{array}{c}\log _{10} \\
\text { (age/yr) }\end{array}$ & $m_{K s}^{\mathrm{b}}$ \\
\hline N33_24311-D & 1 & $12: 37: 06.55$ & $62: 15: 35.4$ & 25.3 & 3.474 & $1.1_{-0.3}^{+0.2}$ & $15.8_{-0.6}^{+1.0}$ & $0.05_{-0.00}^{+0.00}$ & $7.91_{-0.15}^{+0.10}$ & $24.1 \pm 0.2$ \\
\hline N33_19880-D & 1 & $12: 36: 55.14$ & $62: 15: 29.2$ & 26.7 & 3.405 & $1.4_{-1.2}^{+2.8}$ & $3.4_{-1.3}^{+15.6}$ & $0.05_{-0.05}^{+0.10}$ & $8.71_{-1.71}^{+0.55}$ & $25.1 \pm 0.4$ \\
\hline N33_25713-D & 1 & $12: 37: 10.62$ & $62: 14: 52.6$ & 25.6 & 3.615 & $5.7_{-3.2}^{+3.2}$ & $37.2_{-15.5}^{+40.0}$ & $0.20_{-0.05}^{+0.05}$ & $8.26_{-0.70}^{+0.45}$ & $24.5 \pm 0.2$ \\
\hline N33_18549-D & 1 & $12: 36: 51.89$ & $62: 15: 14.5$ & 26.0 & 3.333 & $6.5_{-0.5}^{+3.0}$ & $21.8_{-8.6}^{+0.5}$ & $0.20_{-0.05}^{+0.00}$ & $8.56_{-0.05}^{+0.40}$ & $24.3 \pm 0.2$ \\
\hline N33_19374-D & 1 & $12: 36: 53.88$ & $62: 14: 18.0$ & 26.3 & 3.653 & $78.6_{-19.5}^{+11.2}$ & $70.7_{-1.2}^{+3.2}$ & $0.35_{-0.00}^{+0.00}$ & $9.16_{-0.15}^{+0.07}$ & $23.9 \pm 0.1$ \\
\hline N33_24278-D & 1 & $12: 37: 06.46$ & $62: 13: 20.5$ & 25.8 & 3.671 & $6.7_{-4.8}^{+2.5}$ & $7.5_{-0.1}^{+6.1}$ & $0.05_{-0.00}^{+0.05}$ & $9.06_{-0.85}^{+0.15}$ & $25.2 \pm 0.5$ \\
\hline N33_25726-D & 1 & $12: 37: 10.66$ & $62: 12: 39.0$ & 25.2 & 3.733 & $10.4_{-1.9}^{+2.3}$ & $39.2_{-0.8}^{+0.9}$ & $0.15_{-0.00}^{+0.00}$ & $8.51_{-0.10}^{+0.10}$ & $24.0 \pm 0.1$ \\
\hline N32_20647-D & 1 & $12: 36: 57.00$ & $62: 11: 51.0$ & 26.3 & 3.740 & $0.9_{-0.7}^{+3.5}$ & $9.6_{-6.4}^{+19.4}$ & $0.10_{-0.10}^{+0.05}$ & $8.06_{-1.06}^{+1.15}$ & $25.6 \pm 0.9$ \\
\hline N32_23933-D & 1 & $12: 37: 05.52$ & $62: 11: 27.2$ & 25.3 & 3.469 & $11.8_{-1.0}^{+1.0}$ & $106.8_{-2.8}^{+2.7}$ & $0.30_{-0.00}^{+0.00}$ & $8.11_{-0.05}^{+0.05}$ & $23.4 \pm 0.1$ \\
\hline N32_15359 & 1 & $12: 36: 43.54$ & $62: 11: 21.4$ & 24.4 & 3.488 & $11.7_{-0.7}^{+1.8}$ & $1047.6_{-122.6^{+74.4}}^{\mathrm{a}}$ & $0.40_{-0.00}^{+0.00}$ & $7.06_{-0.06}^{+0.12}$ & $23.0 \pm 0.1$ \\
\hline N42_7697-D & 2 & $12: 36: 22.17$ & $62: 09: 42.3$ & 25.8 & 3.771 & $2.1_{-1.6}^{+1.4}$ & $9.9_{-0.4}^{+42.1}$ & $0.05_{-0.00}^{+0.10}$ & $8.41_{-1.41}^{+0.25}$ & $25.5 \pm 0.7$ \\
\hline N42_12130-D & 2 & $12: 36: 35.15$ & 62:08:50.8 & 26.0 & 3.474 & $1.7_{-0.3}^{+1.6}$ & $171.7_{-107.3}^{+0.0}$ & $0.35_{-0.05}^{+0.00}$ & $7.00_{-0.00}^{+0.76}$ & $25.5 \pm 0.7$ \\
\hline N42_11065-D & 2 & $12: 36: 32.24$ & $62: 09: 46.5$ & 25.4 & 3.658 & $2.1_{-0.8}^{+0.2}$ & $20.8_{-0.4}^{+20.8}$ & $0.10_{-0.00}^{+0.05}$ & $8.06_{-0.54}^{+0.05}$ & $25.1 \pm 0.4$ \\
\hline N32_14225 & 2 & $12: 36: 40.58$ & $62: 10: 41.1$ & 26.0 & 3.245 & $11.7_{-5.1}^{+7.2}$ & $49.2_{-19.7}^{+42.9}$ & $0.30_{-0.05}^{+0.05}$ & $8.46_{-0.55}^{+0.45}$ & $24.1 \pm 0.2$ \\
\hline N32_16805 & 2 & $12: 36: 47.57$ & $62: 10: 23.7$ & 25.9 & 3.235 & $7.6_{-0.6}^{+0.0}$ & $69.0_{-0.0}^{+2.2}$ & $0.30_{-0.00}^{+0.00}$ & $8.11_{-0.05}^{+0.00}$ & $23.9 \pm 0.1$ \\
\hline N32_15430 & 2 & $12: 36: 43.79$ & $62: 11: 20.0$ & 25.8 & 3.237 & $3.8_{-0.5}^{+2.5}$ & $272.7_{-151.6}^{+45.4}$ & $0.40_{-0.05}^{+0.00}$ & $7.16_{-0.14}^{+0.60}$ & $23.9 \pm 0.1$ \\
\hline N32_19795 & 2 & $12: 36: 54.94$ & $62: 11: 43.8$ & 25.1 & 2.976 & $16.7_{-1.3}^{+0.0}$ & $168.6_{-0.0}^{+5.1}$ & $0.35_{-0.00}^{+0.00}$ & $8.06_{-0.05}^{+0.00}$ & $23.2 \pm 0.1$ \\
\hline N33_23907-D & 2 & $12: 37: 05.48$ & $62: 12: 37.1$ & 25.8 & 3.678 & $2.2_{-1.7}^{+2.6}$ & $11.6_{-4.9}^{+48.5}$ & $0.10_{-0.05}^{+0.10}$ & $8.36_{-1.36}^{+0.60}$ & $25.1 \pm 0.4$ \\
\hline N33_20428 & 2 & $12: 36: 56.48$ & $62: 13: 39.9$ & 26.8 & 3.436 & $2.9_{-2.5}^{+4.3}$ & $7.8_{-3.1}^{+57.5}$ & $0.15_{-0.05}^{+0.15}$ & $8.66_{-1.66}^{+0.60}$ & $24.6 \pm 0.3$ \\
\hline N33_18453 & 2 & $12: 36: 51.69$ & $62: 15: 10.1$ & 25.8 & 3.364 & $0.2_{-0.0}^{+0.1}$ & $25.0_{-6.8}^{+0.0}$ & $0.10_{-0.00}^{+0.00}$ & $7.00_{-0.00}^{+0.34}$ & $24.4 \pm 0.2$ \\
\hline N32_18454 & 1 & $12: 36: 51.69$ & $62: 12: 15.1$ & 26.5 & $3.88_{-0.25}^{+0.17}$ & $1.2_{-0.4}^{+0.5}$ & $2.9_{-0.3}^{+4.5}$ & $0.00_{-0.00}^{+0.10}$ & $8.71_{-0.55}^{+0.15}$ & $<26.3$ \\
\hline N42_7643 & 2 & $12: 36: 22.00$ & $62: 09: 12.7$ & 26.5 & $2.70_{-0.20}^{+0.10}$ & $4.3_{-0.9}^{+1.3}$ & $169.1_{-102.8}^{+31.7}$ & $0.50_{-0.05}^{+0.00}$ & $7.44_{-0.12}^{+0.52}$ & $24.1 \pm 0.2$ \\
\hline N42_10036 & 2 & $12: 36: 29.20$ & $62: 08: 53.5$ & 27.3 & $2.92_{-0.19}^{+0.21}$ & $5.8_{-2.7}^{+9.0}$ & $5.8_{-3.6}^{+21.8}$ & $\begin{array}{l}0.20_{-0.10}^{+0.15} \\
\text { - }\end{array}$ & $9.11_{-0.90}^{+0.84}$ & $25.0 \pm 0.4$ \\
\hline N42_10800 & 2 & $12: 36: 31.53$ & $62: 10: 06.4$ & 27.2 & $2.92_{-0.30}^{+1.06}$ & $0.1_{-0.0}^{+0.1}$ & $7.2_{-5.8}^{+5.1}$ & $0.15_{-0.15}^{+0.05}$ & $7.00_{-0.00}^{+0.96}$ & $<26.3$ \\
\hline N32_17776 & 2 & $12: 36: 50.09$ & $62: 10: 23.7$ & 26.0 & $3.54_{-0.24}^{+0.20}$ & $1.5_{-1.0}^{+2.6}$ & $26.7_{-13.6}^{+53.2}$ & $0.20_{-0.05}^{+0.05}$ & $7.81_{-0.81}^{+0.75}$ & $25.4 \pm 0.7$ \\
\hline N42_13119 & 2 & $12: 36: 37.65$ & $62: 12: 10.8$ & 27.1 & $3.13_{-0.54}^{+0.60}$ & $0.5_{-0.3}^{+1.4}$ & $26.1_{-20.5}^{+37.3}$ & $0.30_{-0.10}^{+0.05}$ & $7.26_{-0.26}^{+1.25}$ & $<26.3$ \\
\hline N32_15794 & 2 & $12: 36: 44.77$ & $62: 11: 45.8$ & 26.9 & $3.54_{-0.29}^{+0.20}$ & $1.1_{-1.0}^{+5.6}$ & $1.9_{-0.3}^{+14.6}$ & $0.00_{-0.00}^{+0.15}$ & $8.86_{-1.86}^{+0.84}$ & $<25.6$ \\
\hline N32_18900 & 2 & $12: 36: 52.71$ & $62: 11: 37.3$ & 25.9 & $3.54_{-0.13}^{+0.24}$ & $5.3_{-1.2}^{+1.2}$ & $66.5_{-26.6}^{+12.7}$ & $0.30_{-0.05}^{+0.00}$ & $7.96_{-0.15}^{+0.25}$ & $24.8 \pm 0.3$ \\
\hline
\end{tabular}

Notes. Sample IDs, coordinates, redshifts, and properties derived from SED fitting. Where appropriate, the emission line flux has been subtracted from the $K_{S}$-band photometry before fitting to the photometry, using only the stellar continuum to determine SED properties. Mask "1" denotes objects observed on our March 20-21 mask "2" denotes those undertaken April 16-17. This table contains the full list of 28 objects we observed, with the 20 galaxies with secure spectroscopic confirmation listed first. The identifier "-D" denotes objects with previously known spectroscopic redshifts from our DEIMOS campaign.

${ }^{a}$ When the SFR for this object is estimated using the dust correction from Meurer et al. (1999) and the UV continuum to SFR conversion of Kennicutt (1998), we find a much more modest rate of $150 M_{\odot} \mathrm{yr}^{-1}$.

b Total measured $K_{S}$ magnitude, including any line emission.

\section{ANALYSIS}

The primary goal of this work is to verify or otherwise refute the conclusions of Stark et al. (2013), who examined the EW distribution for $\mathrm{H} \alpha$ emission for LBGs of known spectroscopic redshift in the range $3.8<z<5.0$. A significant conclusion from this study was the remarkably strong emission deduced by SED fitting. Using near-infrared spectroscopy with MOSFIRE, not only can we directly measure the EW distribution of [O III] emission, but we can also test the robustness of the SED-fitting approach by comparing spectroscopic line fluxes with those inferred from broadband photometry.

\subsection{Equivalent Width Distribution}

Even a cursory inspection of our MOSFIRE spectra revealed the presence of many intense line emitters. Figure 2 shows the 2D spectra for four targets showing strong emission where the continuum remains undetected. To assemble the EW distribution, we considered all objects with a spectroscopic detection in either our DEIMOS or MOSFIRE campaigns (Table 1). Our sample of 20 objects spans $2.97 \leqslant z \leqslant 3.77$ with a median $z=3.47$. To determine the continuum level necessary to measure the EW, we corrected the $K$-band photometry for the observed fluxes of any emission lines seen in the MOSFIRE spectra. Where one of the [O III] doublet lines was partially or fully obscured by a sky line, we assumed its flux adopting a fixed 5007/4959 line ratio of 3.0. For our two spectroscopic targets for which no significant line fluxes were detected in our MOSFIRE data, we derived $1 \sigma$ upper limits for each nebular line EW. The photometry for one of our targets, N33_19374, is likely contaminated by a nearby object. In this case, we made no attempt to correct for the flux falling inside our apertures from other objects, but estimate the EW as a lower limit.

EWs and errors were measured using a Bayesian Monte Carlo technique. Because our EWs depend on the measured line fluxes both directly and indirectly through the subtraction of the emission component from the $K_{S}$-band photometry, accurate errors are non-trivial and can be asymmetric. To account for this, 

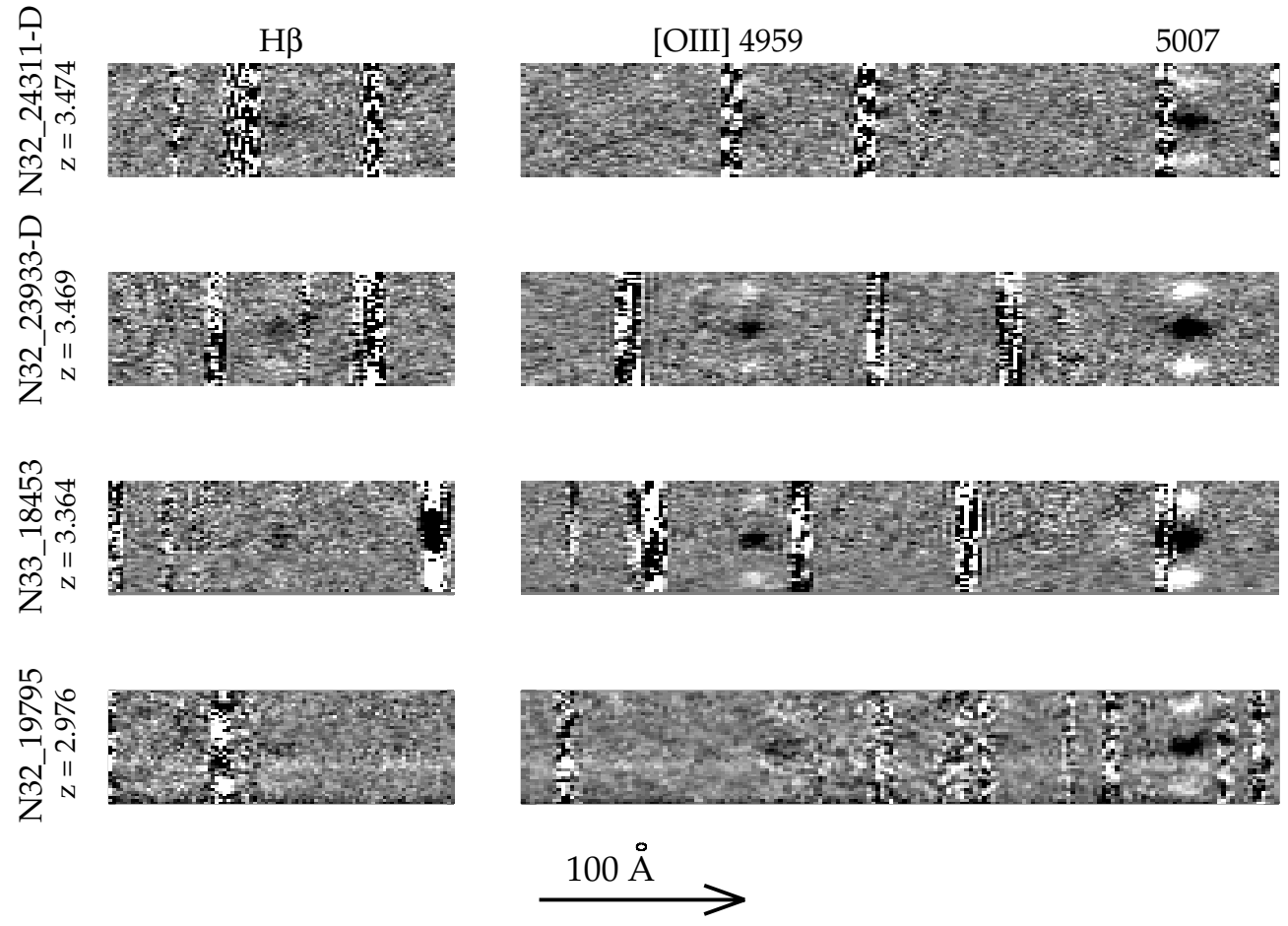

Figure 2. Example 2D MOSFIRE spectra in A-B-A format for four targets showing prominent nebular emission. The left panels focus on the region containing H $\beta$ and the right panels focus on the [O III] doublet (marked).
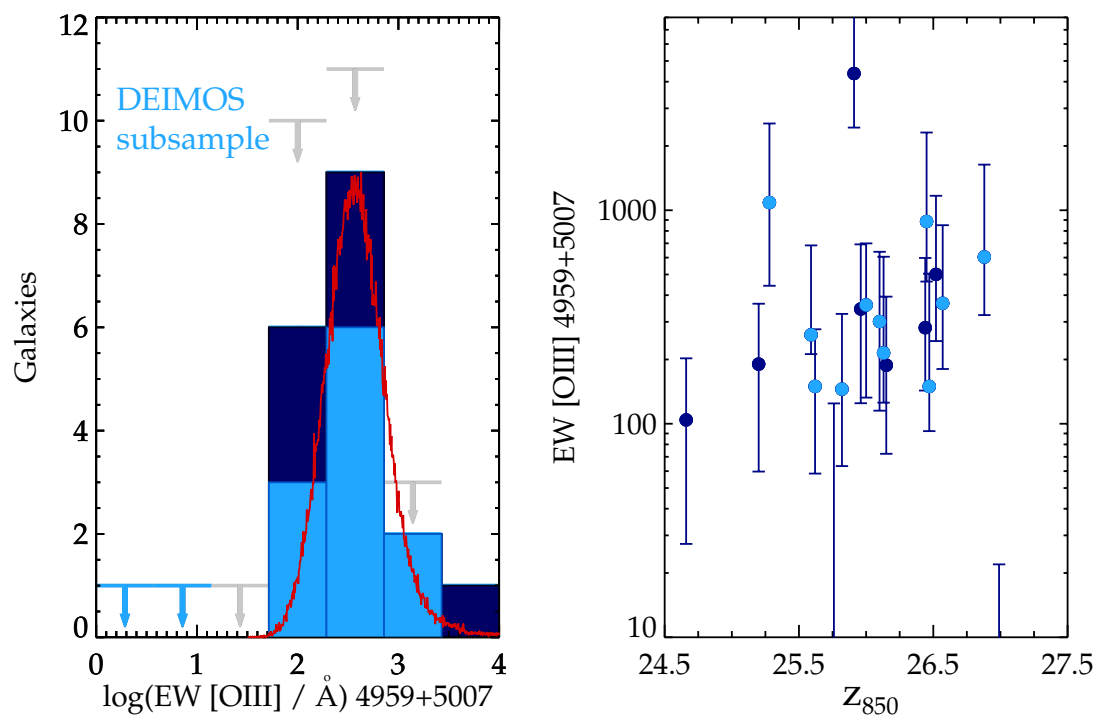

Figure 3. Left: the rest-frame equivalent width distribution of [O III] $4959+5007 \AA$ A derived from our MOSFIRE spectroscopic data. The red curve estimates the distribution expected from the distribution for $\mathrm{H} \alpha$ derived from SED fitting method by Stark et al. (2013). The light blue downward pointing arrows indicate secure upper limits from our DEIMOS sample with previously known redshifts. The gray downward pointing arrows indicate upper limits for the eight objects in our photometric sample that display no strong lines, but which may not lie in the required $3.0<z<3.8$ redshift range. Right: individual equivalent widths of [O III] vs. z 850 magnitude. In both panels, light blue denotes the spectroscopic sample and dark blue denotes the photometric sample (see Section 2).

(A color version of this figure is available in the online journal.)

we run a Monte Carlo simulation with $N=10000$ trials for each galaxy. This simulation takes the actual measured line fluxes and $K_{S}$ photometry, perturbs each by the appropriate error, and then calculates the appropriate continuum magnitude and EWs. At each step, we apply a prior that the fluxes must not be negative to ensure a distribution that reflects reality. For any galaxies with a line flux measured at less than $1 \sigma$, we instead calculate a $1 \sigma$ upper limit in the same manner, given by the smallest EW greater than the value of $68 \%$ of our Monte Carlo trials.
Table 2 presents the spectroscopic line measures and the restframe EW distribution of [O III] $4959+5007$ is presented in Figure 3. It is immediately clear that most have very intense emission lines with a median [O III] $\mathrm{EW}_{\text {rest }}$ of $280 \AA$ A. There is a significant tail to much higher values; two galaxies have $\mathrm{EW}_{\text {rest }}>1000 \AA$, where the $K_{S}$-band photometry is dominated by line emission. Importantly, we see no significant difference in the distribution for those galaxies selected on the basis of their DEIMOS spectroscopy and those photometrically selected entirely for this study. 
Table 2

MOSFIRE Spectroscopic Measurements

\begin{tabular}{|c|c|c|c|c|c|c|}
\hline ID & $\begin{array}{c}\text { flux }_{\mathrm{H} \beta} \\
\left(10^{-18} \mathrm{erg} \mathrm{cm}^{-1} \mathrm{~s}^{-1}\right)\end{array}$ & $\begin{array}{c}\text { flux }_{[\mathrm{O} \mathrm{III}]^{\mathrm{a}}} \\
\left(10^{-18} \mathrm{erg} \mathrm{cm}^{-1} \mathrm{~s}^{-1}\right)\end{array}$ & $\begin{array}{l}\mathrm{EW}_{\mathrm{H} \beta} \\
(\AA, \text { rest })\end{array}$ & $\begin{array}{l}\mathrm{EW}_{[\mathrm{OIII}]^{\mathrm{a}}} \\
(\AA, \text { rest })\end{array}$ & $\begin{array}{c}v_{\text {neb }}-v_{\text {Ly } \alpha} \mathrm{b} \\
\left(\mathrm{km} \mathrm{s}^{-1}\right)\end{array}$ & $\frac{\text { flu }_{\text {pred,SED }}}{\text { flu }_{\mathrm{obs}}}$ \\
\hline N33_24311-D & $13.5 \pm 2.0$ & $99.5 \pm 3.2$ & $140_{-100}^{+240}$ & $1100_{-200}^{+600}$ & $152 \pm 23$ & 0.91 \\
\hline N33_19880-D & $5.9 \pm 1.7$ & $35.9 \pm 3.9$ & $89_{-35}^{+196}$ & $600_{-200}^{+550}$ & $4 \pm 210$ & $\ldots$ \\
\hline N33_25713-D & $7.2 \pm 2.3$ & $21.7 \pm 4.2$ & $47_{-29}^{+78}$ & $150_{-30}^{+70}$ & $106 \pm 100$ & $\ldots$ \\
\hline N33_18549-D & $22.5 \pm 2.9$ & $38.9 \pm 2.5$ & $170_{-120}^{+240}$ & $300_{-50}^{+100}$ & $319 \pm 60$ & 0.74 \\
\hline N33_19374-D & $-3.0 \pm 2.7$ & $1.1 \pm 4.7$ & $<7$ & $<21$ & $\mathrm{XXX}$ & $\ldots$ \\
\hline N33_24278-D & $-8.6 \pm 3.2$ & $32.8 \pm 6.4$ & $<20$ & $370_{-120}^{+190}$ & $207 \pm 173$ & $\ldots$ \\
\hline N33_25726-D & $9.5 \pm 3.8$ & $79.9 \pm 8.0$ & $40_{-21}^{+61}$ & $360_{-50}^{+60}$ & $86 \pm 45$ & $\ldots$ \\
\hline N32_20647-D & $9.8 \pm 3.6$ & $56.2 \pm 7.3$ & $130_{-40}^{+310}$ & $890_{-320}^{+690}$ & $113 \pm 71$ & $\ldots$ \\
\hline N32_23933-D & $14.0 \pm 2.5$ & $49.1 \pm 3.8$ & $40_{-30}^{+49}$ & $150_{-20}^{+20}$ & $195 \pm 100$ & 2.31 \\
\hline N32_15359 & $11.9 \pm 2.1$ & $54.8 \pm 5.0$ & $21_{-17}^{+26}$ & $100_{-10}^{+10}$ & $\ldots$ & 1.39 \\
\hline N42_7697-D & $7.9 \pm 6.2$ & $18.6 \pm 9.2$ & $91_{-5}^{+226}$ & $260_{-160}^{+220}$ & $227 \pm 98$ & $\ldots$ \\
\hline N42_12130-D & $5.5 \pm 3.3$ & $6.0 \pm 4.4$ & $65_{-7}^{+196}$ & $150_{-90}^{+210}$ & XXX & $\ldots$ \\
\hline N42_11065-D & $2.0 \pm 4.3$ & $1.2 \pm 7.3$ & $<80$ & $<120$ & $\mathrm{xxx}$ & 18.74 \\
\hline N32_14225 & $7.2 \pm 3.5$ & $47.6 \pm 9.0$ & $32_{-18}^{+65}$ & $280_{-80}^{+100}$ & $\ldots$ & $\ldots$ \\
\hline N32_16805 & $20.7 \pm 12.0$ & $39.6 \pm 5.8$ & $79_{-29}^{+162}$ & $190_{-40}^{+50}$ & $\ldots$ & 1.42 \\
\hline N32_15430 & $11.3 \pm 3.7$ & $68.6 \pm 5.2$ & $50_{-31}^{+82}$ & $340_{-50}^{+80}$ & $\ldots$ & 1.01 \\
\hline N32_19795 & $8.4 \pm 6.9$ & $94.2 \pm 7.5$ & $17_{-4}^{+29}$ & $190_{-20}^{+20}$ & $\ldots$ & 1.60 \\
\hline N33_23907-D & $6.0 \pm 4.9$ & $18.3 \pm 6.3$ & $54_{-3}^{+139}$ & $210_{-120}^{+200}$ & $\mathrm{xxx}$ & $\cdots$ \\
\hline N33_20428 & $8.1 \pm 3.2$ & $45.6 \pm 4.9$ & $84_{-32}^{+164}$ & $500_{-130}^{+280}$ & $\ldots$ & $\ldots$ \\
\hline N33_18453 & $9.6 \pm 1.7$ & $126.5 \pm 3.9$ & $320_{-150}^{+860}$ & $4400_{-2000}^{+6200}$ & $\ldots$ & 1.63 \\
\hline
\end{tabular}

Notes. MOSFIRE emission line measurements for our sample.

a Includes contribution from both the 4959 and $5007 \AA$ lines.

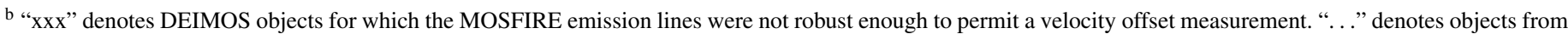
our photometric sample.

\subsection{Comparison with Stark et al. 2013}

Although the foregoing suggests that intense line emission, sufficient to significantly influence the broadband photometry, is quite a common property of $z \simeq 3-4$ LBGs, we now turn to whether or not the EW distribution within the present sample of 20 LBGs supports the conclusions of Stark et al. (2013), who derived the $\mathrm{EW}_{\text {rest }}$ distribution of $\mathrm{H} \alpha$ from a larger sample of 45 LBGs at $3.8<z<5.0$ using SED fitting.

First, it is important to determine whether the LBG samples in the two studies are broadly comparable. In Figure 4, we demonstrate that the UV luminosity distributions of the two samples are fairly similar, with a median absolute magnitude $M_{\mathrm{UV}}=-21.0$ for the Stark et al. (2013) objects compared with $M_{\mathrm{UV}}=-20.0$ for our present sample. Since the UV luminosity correlates closely with the star formation rate and prominence of Lyman $\alpha(\operatorname{Ly} \alpha)$ emission (Stark et al. 2010), this suggests that their nebular emission properties should not be too dissimilar. However, it is important to keep in mind that studies at lower redshift (e.g., Erb et al. 2006; Fumagalli et al. 2012) have found that $\mathrm{H} \alpha \mathrm{EW}$ s increase with decreasing mass (and thus decreasing $M_{\mathrm{UV}}$, through the $M_{*}-M_{\mathrm{UV}}$ relation).

Stark et al. (2013) found that the strength of $\mathrm{H} \alpha$ emission at $z \simeq 4.5$ could be fit well by a $\log$ normal distribution with $\log _{10}\left(\mathrm{EW}_{\text {rest }} / \AA\right)=2.57$ and $\sigma=0.25$. To facilitate a comparison with the present [O III] data, we adopt a value of 2.2 for the flux ratio of [O III] to $\mathrm{H} \alpha$, taken from the empirical compilation of Anders \& Fritze-v. Alvensleben (2003). This ratio is appropriate for a metallicity $Z=0.2 Z_{\odot}$, consistent with that inferred from a stack of LBG spectra at $z \sim 4$ (Jones et al. 2012), as well as measurements of ionized gas in LBGs at
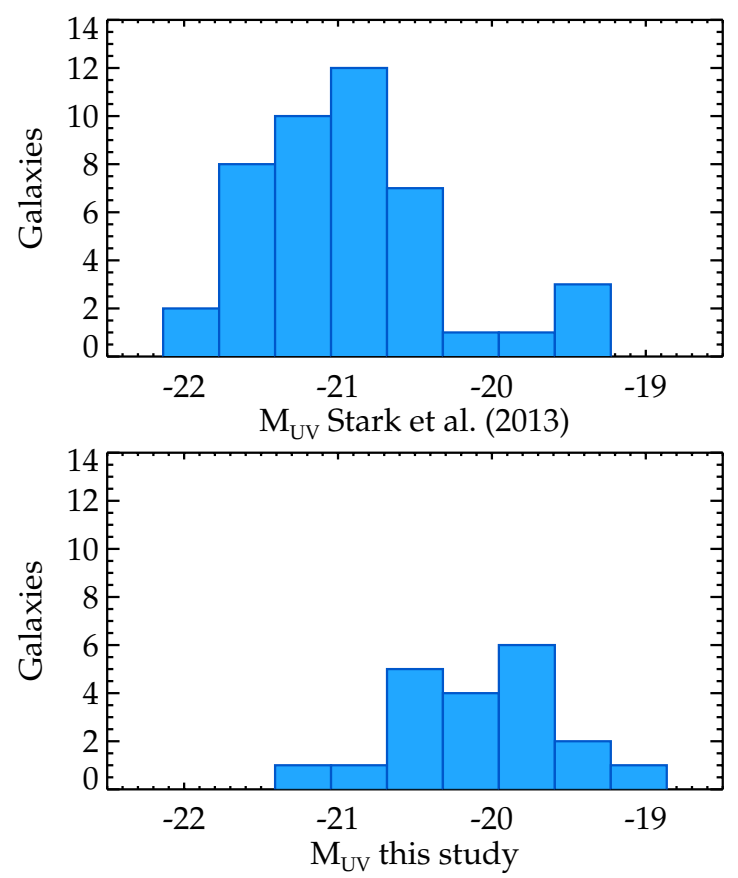

Figure 4. Absolute UV magnitude distribution for the $3.8<z<5.0$ sample discussed by Stark et al. (2013; top panel) compared with that at $3.0<z<3.8$ studied in this paper. Here, we only include the 20 galaxies spectroscopically confirmed to be at $3.0<z<3.8$.

(A color version of this figure is available in the online journal.) 
$z \sim 3.5$ (Maiolino et al. 2008). We caution that this ratio will change slightly with stellar population age, but as most ionizing photons arise from stars with $t<10 \mathrm{Myr}$, such an effect should be very small.

To simulate the expected [O III] $\mathrm{EW}_{\text {rest }}$ distribution, we must also account for noise in both the $K_{S}$-band photometry and the line fluxes, which we incorporate using a Monte Carlo distribution using the uncertainties quoted earlier. The result is the curve in Figure 3, which provides a good fit to the MOSFIRE data and a secure confirmation of large nebular line EWs.

Importantly, we also wish determine the best-fit log-normal distribution to our measurements of [O III] EW. To account for our eight photometrically selected galaxies that exhibit no detectable line emission, noting the uncertainties in the photometric redshifts with respect to the desired range, we consider two extreme cases: (1) all these galaxies lie outside the $3.0<z<3.8$ redshift range, and we can thus ignore them when deriving distribution properties, or (2) all eight galaxies lie within this range, but their line fluxes are below our detection limits. For the second case, we assume that the line EWs are distributed with a flat prior in log space between their derived $1 \sigma$ upper limit (shown as gray downward arrows in Figure 3) and 0 . Assuming case 1 (2), we find best fit parameters of $\log _{10}\left(\mathrm{EW}_{\text {rest }} / \AA\right)=2.4(2.3)$ and $\sigma=0.35(0.5)$. Thus, even in the pessimistic scenario, our spectra still support the strong line emission observed from these sources on average.

Since we also observe the $\mathrm{H} \beta$ line in many of our MOSFIRE spectra, albeit at low significance, this provides an additional check on the expected strength of $\mathrm{H} \alpha$ at these redshifts. To compute the expected $\mathrm{H} \alpha$ flux, we assume a Case $\mathrm{B}$ recombination $\mathrm{H} \alpha$ to $\mathrm{H} \beta$ flux ratio of $2.87: 1$ and compute the differential reddening from the best fit SED for each galaxy assuming a Calzetti reddening law (Calzetti et al. 2000). This yields a predicted $\mathrm{H} \alpha$ flux for each object. The stellar continuum at the location of $\mathrm{H} \alpha$ is likewise estimated from each best-fit SED. To avoid biasing our estimate to only galaxies with strong emission lines, we compute an average $\mathrm{H} \alpha \mathrm{EW}$ for only the 13 galaxies with DEIMOS spectra in our sample and do not weight the values inversely to their errors while averaging. We then find a value of $\mathrm{EW}_{\text {rest }}(\mathrm{H} \alpha)=380 \AA$, providing further support for the strong lines projected by the analysis of Stark et al. (2013).

\subsection{Verifying the SED Fitting Method}

In addition to verifying that our MOSFIRE data of [O III] emission are broadly consistent with the inferences for $\mathrm{H} \alpha$ deduced from SED fitting, we can perform one final check by applying the SED fitting method used by Stark et al. (2013) to the present sample and compare the inferred [O III] fluxes with those measured directly in the near-infrared spectra.

We utilize the Bruzual and Charlot 2007 library of synthetic spectra. For simplicity, we only consider models with a constant star formation history, age $\geqslant 10 \mathrm{Myr}$, and a metallicity of $Z=0.2 Z_{\odot}$, consistent with observations of LBGs at $z \sim 3$ (Shapley et al. 2003). We utilize the Calzetti et al. (2000) prescription for reddening, varying $E(B-V)$ in steps of 0.025 . Synthetic fluxes are calculated for each filter and the $\chi^{2}$ is computed for each model to assess its likelihood.

For all objects with either an IRAC 3.6 or $4.5 \mu \mathrm{m}$ detection, we predict the [O III] line flux using the SED fitting technique adopted by Stark et al. (2013). We fit our grid of stellar continuum models to the observed photometry of each galaxy, excluding the $K$ band. For the best fitting SED, we then compute a synthetic $K$-band flux and determine the emission line strength from the residual. This can only be applied for nine galaxies from Table 1 for which a detection is available in at least one IRAC filter.

We list the ratios of the SED-predicted flux to that actually observed for the nine objects in Table 2. Overall, the results are in good agreement: only one object, N42_11065, is a catastrophic outlier, with an [O III] flux implied from the SED method of 4.0 times our $1 \sigma$ upper limit from the spectrum. The remaining 8 objects all have predicted to observed fluxes within a factor of 2.5 and 7 are within a factor $\leqslant 1.6$. Such an agreement provides a clear validation that the technique we used in Stark et al. (2013) can provide line strength measurements suitable for statistical purposes.

\subsection{Ly $\alpha$ Velocity Offsets}

Since we now possess a sample of $z \simeq 3.5$ galaxies with both optical and near-infrared spectra, we can comment briefly on the prevalence of outflows. As is well known, a velocity offset is often observed between $\operatorname{Ly} \alpha$, which is easily resonantly scattered by hydrogen on its way out of a galaxy, and other nebular lines that trace directly the sites of star formation and provide a systemic redshift, (e.g., Shapley et al. 2003). In particular, Ly $\alpha$ is often observed with a positive velocity offset, suggesting those photons are only able to escape after being scattered by an outflowing $\mathrm{H}_{\mathrm{I}}$ wind on the far side of the galaxy and shifting out of resonance with any $\mathrm{H}$ i on the near side (Steidel et al. 2010).

Measures of this offset velocity at high redshift can shed light on some outstanding issues related to cosmic reionization. First, one of the major currently unknown variables that enters into reionization calculations is $f_{\text {esc }}$, the escape fraction of ionizing photons from galaxies. Although recent measurements have found $f_{\text {esc }} \simeq 10 \%$ at $z \sim 3$ (Nestor et al. 2013), direct measurements are impossible at higher redshifts, owing to the increased opacity of the intergalactic medium (IGM). A higher value $(\simeq 20 \%)$ is required to reproduce measurements of the IGM neutral fraction at high redshifts (e.g., Robertson et al. 2013; Kuhlen \& Faucher-Giguère 2012). As any outflowing neutral gas will serve to extinguish ionizing radiation, an observed decrease in the velocity offset of Ly $\alpha$, potentially indicating a lower covering fraction of neutral gas, would provide further support for an increased $f_{\text {esc }}$. The velocity offset is also of direct interest, as numerous experiments seeking to directly probe the ionization state of the IGM at $z>6$ utilize the visibility of Ly $\alpha$ emission (e.g., Pentericci et al. 2011; Schenker et al. 2012; Ono et al. 2012). If $\operatorname{Ly} \alpha$ escapes galaxies with a smaller velocity offset than previously believed, it is closer to resonance and more easily quenched by a neutral IGM.

To this end, we present the difference in the observed velocities of $\mathrm{Ly} \alpha$ and the $\mathrm{H} \beta+[\mathrm{O}$ III] for all objects with at least a $3 \sigma$ line detection in Table 2 and Figure 5. The left panel displays both stacked $\mathrm{H} \beta+[\mathrm{O}$ III $]$ (black) and Ly $\alpha$ (red) profiles, demonstrating the high-fidelity velocity measurements we are able to make with MOSFIRE. In the right panel, we present a compilation of Ly $\alpha$ velocity offset measurements, plotted as a function of redshift and Ly $\alpha \mathrm{EW}_{\text {rest }}$.

As all of the nine targets in our MOSFIRE sample show significant Ly $\alpha$ in emission $(\mathrm{EW}>20 \AA$ ), we must be careful not to draw conclusions by blindly comparing this to the Steidel et al. (2010) sample at lower redshift, for which all objects have only modest EWs. A more illuminating conclusion can perhaps be drawn by compiling the velocity offsets for other galaxies with strong Ly $\alpha$ emission, drawn here from McLinden et al. 

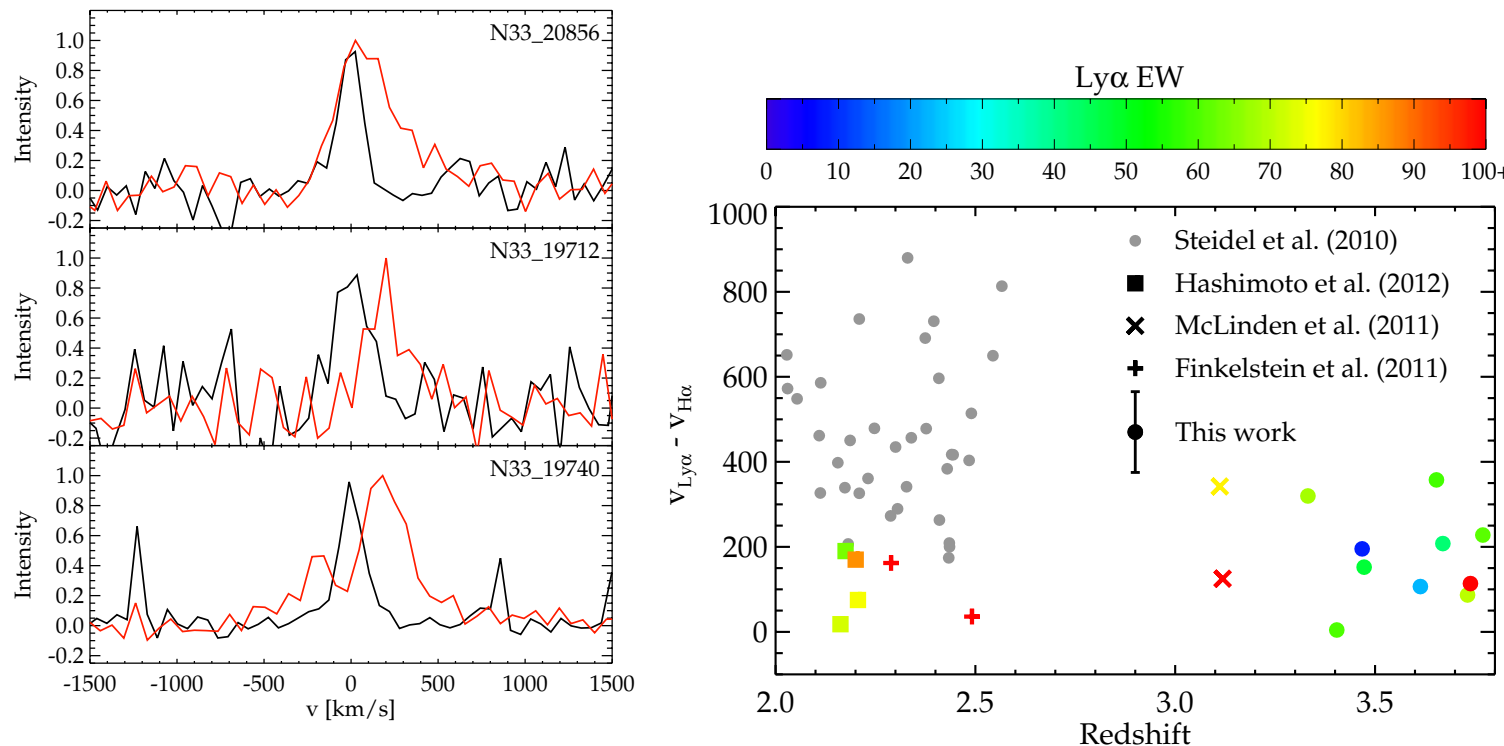

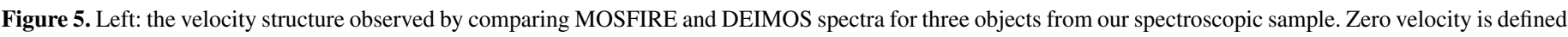

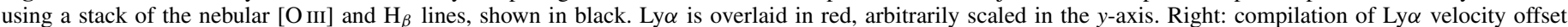

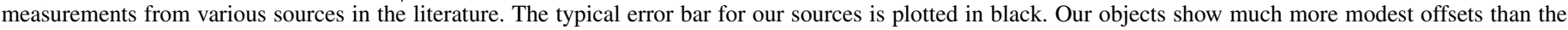

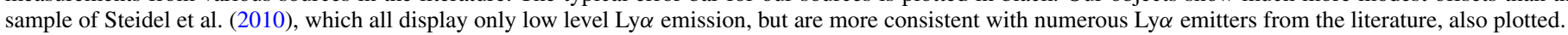

(A color version of this figure is available in the online journal.)

(2011), Finkelstein et al. (2011), and Hashimoto et al. (2013). All have quite low offsets, with $\left\langle v_{\mathrm{Ly} \alpha}\right\rangle=+149 \mathrm{~km} \mathrm{~s}^{-1}$ for the entire sample of 17 objects, and $\left\langle v_{\mathrm{Ly} \alpha}\right\rangle=+157 \mathrm{~km} \mathrm{~s}^{-1}$ for our own 9 objects. As the fraction of star-forming galaxies displaying strong Ly $\alpha$ emission increases with redshift out to at least $z \sim 6$ (Stark et al. 2010), these data imply it may become easier for ionizing photons to escape if this correlation of large $\mathrm{EW}_{\mathrm{Ly} \alpha}$ with $v_{\mathrm{Ly} \alpha}$ trend is in fact caused by lower H I covering fractions.

We note here that a number of other recent papers support the association of larger Ly $\alpha$ EWs with lower covering fractions of neutral gas at high redshift. Hashimoto et al. (2013) find that for a stacked sample of four $z \sim 2$ Ly $\alpha$ emitters, Ly $\alpha$ emerges redshifted from the systemic velocity by approximately the same amount as low ionization absorption lines are blueshifted by. This is in contrast with the theoretical predictions of Verhamme et al. (2006), which predict that such small velocity offsets will only occur for small $\mathrm{H}_{\mathrm{I}}$ column densities $\left(N_{\mathrm{H}}<\right.$ $10^{20}$ ). Additionally, Jones et al. (2013) explicitly derived the minimum covering fraction of neutral gas for a sample of lensed $z \sim 2-4$ LBGs with high resolution spectra and found that this inversely correlates with the $\operatorname{Ly} \alpha \mathrm{EW}$. By virtue of our vastly expanded sample here, doubling the number of LBGs for which simultaneous measurements of $\operatorname{Ly} \alpha$ and rest-frame optical nebular lines are available, we have provided further evidence of the important role the neutral gas covering fraction may play in Ly $\alpha$ escape.

\section{DISCUSSION}

We have shown, through near-infrared spectroscopy with MOSFIRE, that the main conclusions of our earlier work (Stark et al. 2013) are confirmed. A significant fraction of our 20 LBG targets show intense [O III] line emission and the EW distribution is broadly comparable with that inferred for $\mathrm{H} \alpha$ from SED fitting for a larger sample. Moreover, where we can make a direct comparison within our own sample, the SED fitting method predicts [O III] line fluxes that are in reasonable agreement, given the uncertainties, with those measured directly with MOSFIRE.
One might worry that because our DEIMOS-confirmed objects mostly display large Ly $\alpha$ EWs (a result of following up only secure confirmations with MOSFIRE), we are biasing our sample toward especially strong emitters. However, our additional photometric sample of seven objects dissuades this notion. Although the samples remain modest in size, not only does the EW distribution appear similar (Figure 3), but our largest EW object, N33_18453, is also part of this photometric sample. Without optical spectra for these objects, we cannot completely rule out that these galaxies do not all display strong Ly $\alpha$, but our prior work provides a statistical argument that this is not the case. Using the probabilities derived in Stark et al. (2011) that $z \sim 4$ LBGs to exhibit strong $\left(\mathrm{EW}_{\text {rest }}>25 \AA\right.$ ) Ly $\alpha$, we only expect $\sim 2.0$ galaxies in our photometric sample of seven confirmed galaxies.

We can also examine, for our present sample, how our measured line emission affects the derived physical properties. Figure 6 presents SEDs for a selection of our sample where the excess flux in the $K_{S}$ band is clearly visible. We can fit the SEDs using both the entire photometric dataset, including the line-contaminated $K_{S}$ filter, as well as that excluding the contaminated band. In both cases, we assume a stellar continuum only. In this trial, the median properties of the sample hardly change; there is no significant change in age and the stellar mass is reduced by only $3 \%$ when correcting for line emission. This is because the IRAC 3.6 and $4.5 \mu \mathrm{m}$ photometry provides a crucial measurement free from line contamination longward of the Balmer break. Thus, the majority of existing measurements of SED-derived properties of LBGs at $z \sim 3$ that incorporate IRAC data should not be significantly affected by [O III] emission, even though it is particularly intense.

A more illuminating test applies when the IRAC photometry is ignored, in which case the $K_{S}$ filter becomes the only photometric measurement beyond the Balmer break. This is a more appropriate test of how SEDs are fit at $z \sim 6-7$, where both the 3.6 and $4.5 \mu \mathrm{m}$ IRAC filters are contaminated by [O III] and $\mathrm{H} \alpha$, respectively. In this comparison, the implications of line emission are much more striking. The median mass for the line 

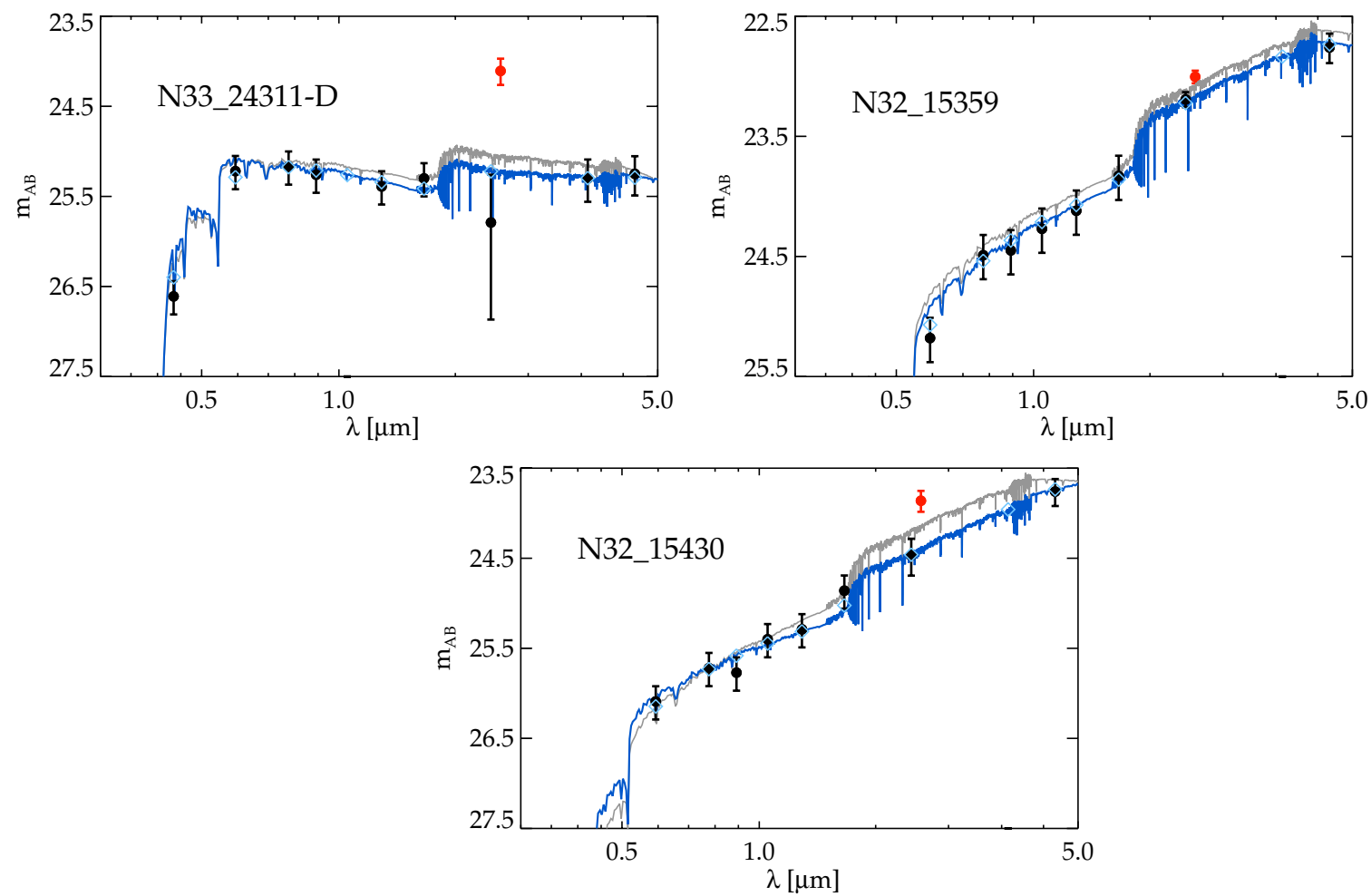

Figure 6. SEDs for three of our targets. The red data point represents the observed $K$-band photometry without correction for [O III] contamination and the gray spectrum shows the best fit SED to these data. The black $K$-band data point shows stellar continuum flux after correction for the MOSFIRE-determined [O III] line flux and the blue spectrum is the associated best fit SED.

(A color version of this figure is available in the online journal.)

corrected SEDs is only $64 \%$ of that of the mass determined using the contaminated photometry and the median age is decreased by $30 \%$. For the most intense emitters, N33_24311 and N33_18453, the masses can be reduced by factors of $\simeq 20$.

We have demonstrated here, for the first time with both robust spectroscopy and significant sample sizes, the strength of nebular emission in $z \geqslant 3$ LBGs, as well as significantly increased the number of the same galaxies with measurements of Ly $\alpha$ velocity offsets. The implications of such observations are extremely important for the high-redshift universe. SED fitting at high redshifts must account for contamination of broadband filters by these strong lines to determine accurate stellar masses. These lines provide mounting evidence for a continued increase of the sSFR beyond $z=2$, which has only recently been suggested. The measurements of precise offsets also provide valuable input to models which seek to map out the universal neutral fraction through $\mathrm{Ly} \alpha$ radiative transfer, and also bolster arguments for an increasing escape fraction with redshift. With the era of multi-object, near-infrared spectrographs just now beginning, prospects for further solidification of these trends will be strong.

We thank Chuck Steidel and Ian McLean for their hard work in developing the MOSFIRE instrument and the anonymous referee for comments that helped significantly improve this manuscript. The Keck observatory staff proved invaluable and we thank them for their dedication to maintaining a world-class observatory. We also wish to recognize and acknowledge the very significant cultural role and reverence that the summit of Mauna Kea has always had within the indigenous Hawaiian community. We are most fortunate to have the opportunity to conduct observations from this mountain. N.P.K. was supported by NSF grant 1106171 .

\section{REFERENCES}

Anders, P., \& Fritze-v. Alvensleben, U. 2003, A\&A, 401, 1063

Atek, H., Siana, B., Scarlata, C., et al. 2011, ApJ, 743, 121

Bertin, E., Mellier, Y., Radovich, M., et al. 2002, in ASP Conf. Proc. 281, Astronomical Data Analysis Software and Systems XI, ed. D. A. Bohlender, D. Durand, \& T. H. Handley (San Francisco, CA: ASP), 228

Calzetti, D., Armus, L., Bohlin, R. C., et al. 2000, ApJ, 533, 682

Coe, D., Benítez, N., Sánchez, S. F., et al. 2006, AJ, 132, 926

Davé, R., Finlator, K., \& Oppenheimer, B. D. 2012, MNRAS, 421, 98 de Barros, S., Schaerer, D., \& Stark, D. P. 2012, arXiv:1207.3663

Erb, D. K., Steidel, C. C., Shapley, A. E., et al. 2006, ApJ, 647, 128

Faber, S. M., Phillips, A. C., Kibrick, R. I., et al. 2003, Proc. SPIE, 4841, 1657

Finkelstein, S. L., Hill, G. J., Gebhardt, K., et al. 2011, ApJ, 729, 140

Fumagalli, M., Patel, S. G., Franx, M., et al. 2012, ApJL, 757, L22

Giavalisco, M., Ferguson, H. C., Koekemoer, A. M., et al. 2004, ApJL, 600, L93

González, V., Bouwens, R., llingworth, G., et al. 2012, arXiv:1208.4362

González, V., Labbé, I., Bouwens, R. J., et al. 2010, ApJ, 713, 115

González, V., Labbé, I., Bouwens, R. J., et al. 2011, ApJL, 735, L34

Grogin, N. A., Kocevski, D. D., Faber, S. M., et al. 2011, ApJS, 197, 35

Hashimoto, T., Ouchi, M., Shimasaku, K., et al. 2013, ApJ, 765, 70

Jones, T., Ellis, R. S., Schenker, M. A., \& Stark, D. P. 2013, ApJ, in press (arXiv:1304.7015)

Jones, T., Stark, D. P., \& Ellis, R. S. 2012, ApJ, 751, 51

Kennicutt, R. C., Jr. 1998, ARA\&A, 36, 189

Koekemoer, A. M., Faber, S. M., Ferguson, H. C., et al. 2011, ApJS, 197, 36 Krueger, H., Fritze-v. Alvensleben, U., \& Loose, H.-H. 1995, A\&A, 303, 41

Kuhlen, M., \& Faucher-Giguère, C.-A. 2012, MNRAS, 423, 862

Labbé, I., González, V., Bouwens, R. J., et al. 2010, ApJL, 716, L103

Maiolino, R., Nagao, T., Grazian, A., et al. 2008, A\&A, 488, 463

McLean, I. S., Steidel, C. C., Epps, H. W., et al. 2012, Proc. SPIE, 8446

McLinden, E. M., Finkelstein, S. L., Rhoads, J. E., et al. 2011, ApJ, 730, 136

McLure, R. J., Dunlop, J. S., de Ravel, L., et al. 2011, MNRAS, 418, 2074

Meurer, G. R., Heckman, T. M., \& Calzetti, D. 1999, ApJ, 521, 64

Nestor, D. B., Shapley, A. E., Kornei, K. A., Steidel, C. C., \& Siana, B. 2013, ApJ, 765,47

Oke, J. B., \& Gunn, J. E. 1983, ApJ, 266, 713

Ono, Y., Ouchi, M., Mobasher, B., et al. 2012, ApJ, 744, 83 
Ono, Y., Ouchi, M., Shimasaku, K., et al. 2010, ApJ, 724, 1524

Papaderos, P., Izotov, Y. I., Fricke, K. J., Thuan, T. X., \& Guseva, N. G. 1998, A\&A, 338, 43

Pentericci, L., Fontana, A., Vanzella, E., et al. 2011, ApJ, 743, 132

Robertson, B. E., Furlanetto, S. R., Schneider, E., et al. 2013, ApJ, 768, 71

Sargent, W. L. W., \& Searle, L. 1970, ApJL, 162, L155

Schaerer, D., \& de Barros, S. 2009, A\&A, 502, 423

Schaerer, D., \& de Barros, S. 2010, A\&A, 515, A73

Schenker, M. A., Stark, D. P., Ellis, R. S., et al. 2012, ApJ, 744, 179

Shapley, A. E., Steidel, C. C., Pettini, M., \& Adelberger, K. L. 2003, ApJ, 588,65
Shim, H., Chary, R.-R., Dickinson, M., et al. 2011, ApJ, 738, 69

Smit, R., et al. 2013, arXiv:1307.5847

Stark, D. P., Ellis, R. S., Bunker, A., et al. 2009, ApJ, 697, 1493

Stark, D. P., Ellis, R. S., Chiu, K., Ouchi, M., \& Bunker, A. 2010, MNRAS, 408, 1628

Stark, D. P., Ellis, R. S., \& Ouchi, M. 2011, ApJL, 728, L2

Stark, D. P., Schenker, M. A., Ellis, R., et al. 2013, ApJ, 763, 129

Steidel, C. C., Erb, D. K., Shapley, A. E., et al. 2010, ApJ, 717, 289

Verhamme, A., Schaerer, D., \& Maselli, A. 2006, A\&A, 460, 397

Wang, W.-H., Cowie, L. L., Barger, A. J., Keenan, R. C., \& Ting, H.-C. 2010, ApJS, 187, 251 